\title{
Economic Evaluation in Duchenne Muscular Dystrophy: Model Frameworks for Cost-Effectiveness Analysis
}

\author{
Erik Landfeldt $^{1} \cdot$ Lars Alfredsson $^{1} \cdot$ Volker Straub $^{2} \cdot$ Hanns Lochmüller $^{2}$ • \\ Katharine Bushby $^{2} \cdot$ Peter Lindgren ${ }^{3,4}$
}

Published online: 31 October 2016

(c) The Author(s) 2016. This article is published with open access at Springerlink.com

\begin{abstract}
Background Several treatments are on the horizon for Duchenne muscular dystrophy (DMD), a terminal orphan disease. In many jurisdictions, decisions regarding pricing and reimbursement of these health technologies comprise evidence of value for money.

Objective The objective of this study was to develop a cost-effectiveness model based on the Duchenne muscular dystrophy Functional Ability Self-Assessment Tool (DMDSAT), a new rating scale created specifically to measure disease progression in clinical practice and trials and model DMD in economic evaluations, and compare it with two alternative model structures.

Methods We constructed three Markov cohort state-transition models to evaluate the cost-effectiveness of a hypothetical intervention for DMD versus standard of care in a UK setting. Model I was based on the DMDSAT,
\end{abstract}

Electronic supplementary material The online version of this article (doi:10.1007/s40273-016-0461-5) contains supplementary material, which is available to authorized users.

Erik Landfeldt

erik.landfeldt@ki.se

1 Institute of Environmental Medicine, Karolinska Institutet, Nobels väg 13, 17177 Stockholm, Sweden

2 Newcastle University John Walton Muscular Dystrophy Research Centre and MRC Centre for Neuromuscular Diseases, Institute of Genetic Medicine, Newcastle University, Newcastle upon Tyne, UK

3 Department of Learning, Informatics, Management and Ethics, Medical Management Centre, Karolinska Institutet, Stockholm, Sweden

4 The Swedish Institute for Health Economics, Lund, Sweden model II on stages of disease as defined in the DMD clinical care guidelines and model III on patients' ventilation status. The conceptual model structures were formulated in collaboration with three DMD experts.

Results All three models were judged to have good validity with regards to the appropriateness of the choice of modelling technique, conceptual representation of the disease, model input data and model outcomes. Across frameworks, lifetime direct medical costs with standard of care ranged between $£ 217,510$ and $£ 284,640$, total costs between $£ 624,240$ and $£ 713,840$, and total number of quality-adjusted life-years between 5.96 and 7.17.

Conclusions We present a first version of a model for the economic evaluation of treatments for DMD based on the DMDSAT, as well as two alternative frameworks encompassing conventional staging of disease progression. Our findings should be helpful to inform health technology assessments and health economic programmes of future treatments for DMD.

\section{Key Points for Decision Makers}

Duchenne muscular dystrophy (DMD) is an orphan disease associated with a substantial burden on affected patients, family caregivers and society.

The Duchenne muscular dystrophy Functional Ability Self-Assessment Tool (DMDSAT) is a new patient-reported outcome scale designed to measure disease progression in DMD.

We present a cost-effectiveness model framework based on the DMDSAT simulating patients across the entire lifetime of disease. 


\section{Introduction}

The exponential growth in health technology innovation during the last decades has realised remarkable medical opportunities. Yet, in a context of financial constraints, the accelerated introduction of health technologies also poses unprecedented challenges for decision makers to regulate uptake and simultaneously manage public expectations of equal access to the most effective, state-of-the-art healthcare. This has been particularly evident for medicines for rare diseases, so-called orphan drugs, which due to small patient populations usually are associated with very high prices to make them commercially viable [1-3].

Following the adoption of legislation to promote research and development of orphan drugs in several jurisdictions, including Australia, the European Union, and the USA, molecules for a wide range of rare illnesses have been licensed or are currently being tested in trials $[4,5]$. As health technology assessment (HTA) of orphan drugs in many countries comprises evidence from economic evaluations, among other factors [6], it has become urgent to better understand the health economic context of specific rare diseases.

An orphan disease for which several compounds soon may be available to patients is Duchenne muscular dystrophy (DMD), a genetic, fatal neuromuscular illness with an incidence of about one in 3800-6300 live male births [7]. DMD is characterised by progressive muscle degeneration resulting in loss of independent ambulation and serious multi-system complications, including cardiac and respiratory failure $[8,9]$. As reported in our previous work, DMD is also associated with a substantial cost burden to society and affected families and significantly impaired health-related quality of life (HR-QOL) (as valued by and compared with the general population) in both patients and caregivers (e.g. parents) [10-12].

The objective of this study was to synthesize our previously published health economic evidence and develop a model framework for the assessment of the cost effectiveness of treatments for DMD based on the Duchenne muscular dystrophy Functional Ability Self-Assessment Tool (DMDSAT) [13], a new rating scale created specifically to measure disease progression in clinical practice and trials and model DMD in economic evaluations. For comparison, we also developed two models based on conventional staging of the disease.

\section{Methods}

\subsection{Model Frameworks}

DMD is a complex disease to model due to heterogeneous presentation with a large number of primary and secondary manifestations [9], for many of which the risk and impact in terms of costs and patient HR-QOL is unknown. This is particularly true for the combined risk and effect of several simultaneous and/or subsequent disease manifestations. Accordingly, instead of modelling a subset of disease-related events for which data will be uncertain, we reasoned that a more robust approach would be to estimate and utilise total cost and HR-QOL estimates-which capture the total health economic impact of DMD-for different stages of the disease. This approach also avoids the risk of having minor complications compete with risks for more serious events (a phenomenon known as 'competing risks'). In addition, modelling stages of disease is in agreement with therapeutic strategies currently being explored for DMD, which aim to slow down the rate of progression (i.e. delay the decline in overall muscle strength and loss of functional ability).

With this in mind, we developed three Markov cohort state-transition models. The conceptual model structures were formulated in collaboration with three internationally renowned DMD experts from Newcastle University (Newcastle upon Tyne, UK): Professor Volker Straub, Professor Hanns Lochmüller and Professor Katharine Bushby (henceforth referred to as DMD experts). Given the grounds for our choice to model stages of disease (described above), we identified no added value in using a model technique incorporating memory at the patient level (e.g. a discrete-event simulation model or a microsimulation model utilising a Markov framework). The models were developed in accordance with the ISPOR-SMDM (International Society for Pharmacoeconomics and Outcomes Research-Society for Medical Decision Making) Modeling Good Research Practices Task Force [14] and designed to evaluate the cost-effectiveness of a hypothetical treatment versus standard of care (SoC) in patients with DMD in a UK setting (Fig. 1).

The framework of model I was based on the DMDSAT [13], a new patient-reported outcome instrument comprising eight questions in four domains (arm function, mobility, transfers and ventilation status) measuring functional ability in patients with DMD on an interval scale ranging from 0 to 23, where higher scores represent higher functional ability. The DMDSAT exhibits excellent psychometric properties and have been shown to have good clinical validity, and is currently the only tool that measures functional ability across the entire trajectory of disease. Model I comprised a total of 25 states, one for each DMDSAT score and an absorbing state for dead.

The framework of model II was based on stages of disease as specified in the international DMD clinical care guidelines [9], defined first in terms of ambulatory status and second in terms of age. It included five states: (1) early ambulatory (approximately age 5-7 years); (2) late 
Fig. 1 The Duchenne muscular dystrophy Markov model frameworks. The absorbing health state 'dead', linked to all states within each model structure, was excluded for simplicity. DMDSAT Duchenne muscular dystrophy Functional Ability Self-Assessment Tool
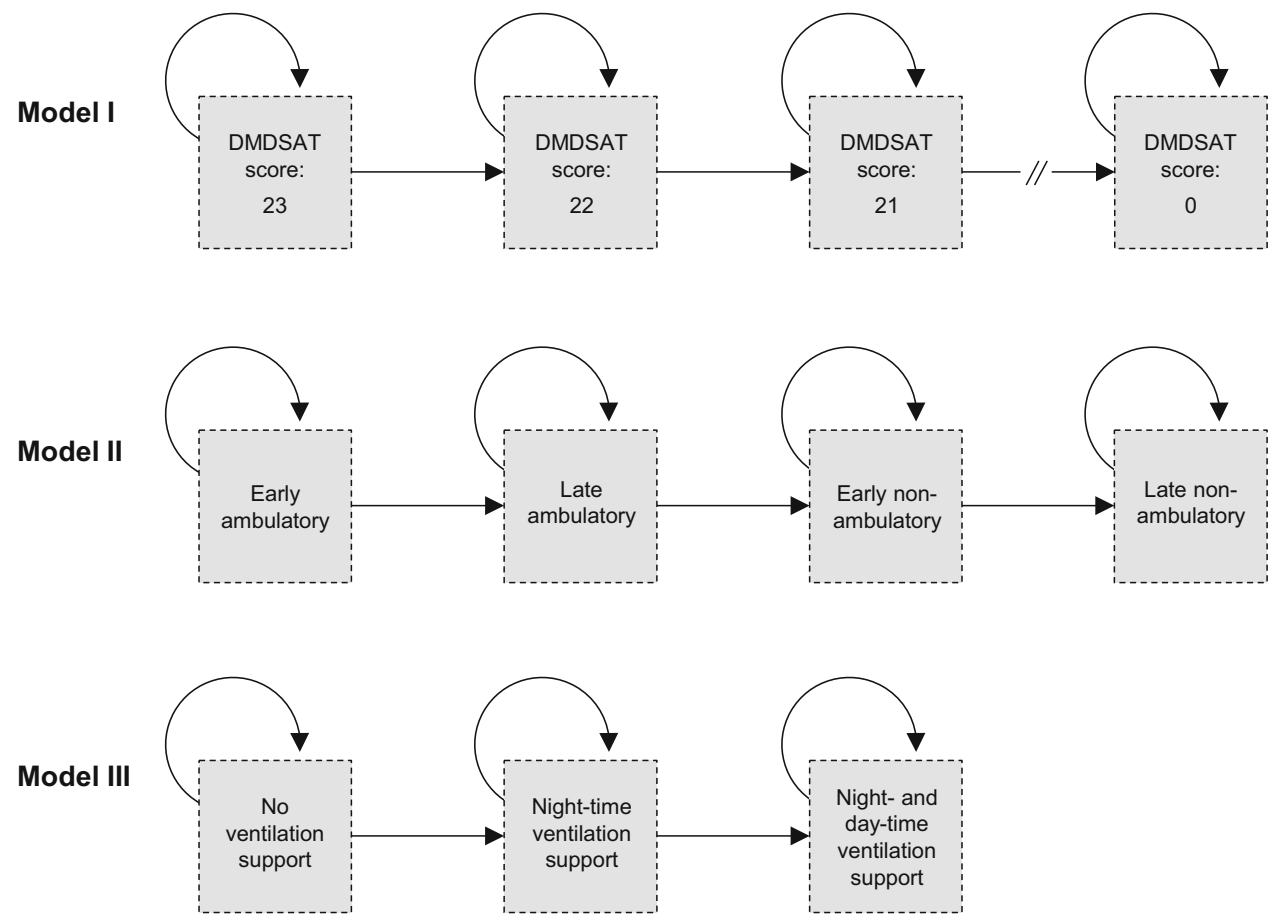

ambulatory (approximately age 8-11 years); (3) early nonambulatory (approximately age 12-15 years); (4) early non-ambulatory (approximately age 16 years or older); and (5) an absorbing state for dead.

Model III was based on patients' ventilation status, which marks key clinical disease milestones and staging for interventions, and comprised four states: (1) no ventilation support; (2) night-time ventilation support; (3) day- and nighttime ventilation support; and (4) an absorbing state for dead.

In each model, every cycle, patients had a probability of remaining in the current state, progressing to a more severe state, or dying, as shown in Fig. 1. For the evaluation results presented in this report, a 12-month cycle was chosen to accommodate the disease progression data (defined in terms of years, described in Sect. 2.2). In model I, we assumed that the cohort started the simulation at a DMDSAT score of 23 (i.e. at full functional ability), in model II in the early ambulatory state, and in model III without ventilation support. All cohorts were followed from the age of 5 years until death (or an age of 100 years). Model input data were collated through a targeted literature review in PubMed and Web of Science (details are provided in the Electronic Supplementary Material Appendix) and from the DMD experts. The models were developed in Microsoft Excel ${ }^{\circledR}$.

\subsection{Progression of Disease}

In model $\mathrm{I}$, in the absence of data, we assumed a linear decline in the rate of progression in terms of DMDSAT scores of two per year, on average. This corresponds to a mean annual probability of $1-\mathrm{e}^{(-2 \times 1)}=0.86$. At this probability, a cohort of patients starting the model simulation at an age of 5 years at a score of 23 (the highest DMDSAT score, corresponding to full functional ability) would be attributed the lowest DMSDAT score $(0$, corresponding to low functional ability) at a mean age of 30 years, identical to the mean age observed in our crosssectional cohort data comprising 770 patients from Germany, Italy, the UK, and the USA [10-13].

In model II, for DMD progression in terms of ambulatory status, we assumed a linear progression based on age (as defined in the DMD clinical care guidelines [9]). Specifically, we assumed that the cohort would, on average, progress between each ambulatory stage every 4 years. Starting the model at an age of 5 years, this corresponds to an annual transition probability of $1-(1-0.5)^{(1 / 4)}=0.16$. At this rate, patients are assumed to become non-ambulatory at an age of 14 years, which is in good agreement with published evidence $[15,16]$ and our clinical observation for patients receiving glucocorticoid treatment.

In model III, state transition probabilities were derived from the mean time to ventilation support. Based on our clinical experience and existing evidence [17], we assumed that patients would require night-time and dayand night-time ventilation support at a mean age of 21 and 28 years, respectively. Starting the model at an age of 5 years, this corresponds to an annual transition probability of $1-(1-0.5)^{(1 / 16)}=0.04$ and $1-(1-0.5)^{(1 /}$ ${ }^{7)}=0.09$, respectively. 


\subsection{Efficacy Data}

To showcase the models, we specified a base-case scenario of a lifelong hypothetical intervention that reduced the probability of disease progression across all model states by a conservative (but realistic) $25 \%$, in agreement with (but in addition to) the efficacy of glucocorticoid treatment observed in clinical practice [15]. For reference, at this efficacy level patients would on average become non-ambulatory at an age of 17 years instead of 14 years (i.e. a mean delay of 3 years). Two alternative treatment durations were explored in sensitivity analysis (described in Sect. 2.8).

\subsection{Cost and Utility Data}

We have previously estimated costs associated with DMD, as well as patient and caregiver HR-QOL, in a cross-sectional, observational study [10-12]. In this previous research, we estimated annual costs of DMD comprising direct medical costs (e.g. hospital admissions, visits to physicians and other healthcare professionals, medical tests and assessments, medications, and emergency and respite care), direct non-medical costs (e.g. costs associated with non-medical aids and investments), informal care costs (i.e. paid and unpaid informal care by the primary caregiver) and indirect costs (i.e. production losses for the patient and primary caregiver due to absenteeism and impaired productivity while working). Details of the cost calculations are provided in the Electronic Supplementary Material Appendix.

Patient HR-QOL was proxy-assessed by the primary caregivers using the Health Utilities Index questionnaire (HUI). The HUI is a generic HR-QOL instrument encompassing 16 questions covering eight dimensions (hearing, speech, ambulation/mobility, pain, dexterity, self-care, emotion and cognition) and has been validated for proxyassessments in ages 5 years and older [18]. Patient utilities were derived using the HUI Mark 3 multi-attribute health status classification system, which is based on preference data collected using the standard gamble method and a visual analogue scale from 256 randomly selected members of the general population in Hamilton, Ontario, Canada [19]. We assessed caregiver HR-QOL using the EuroQol EQ-5D-3L (EQ-5D) [20]. As recommended, EQ$5 \mathrm{D}$ utilities were derived using the UK value set, which is based on preference data collected through the time-tradeoff method from 2997 randomly selected members of the non-institutionalised adult general population in England, Scotland and Wales [21].

These cost and utility data have been mapped to the specified model states in a previous publication [13] by fitting three generalised linear regression models (GLMs) assuming a gamma distribution with a log link as recommended in the literature for skewed, non-censored, nonzero inflated data [22]. The main explanatory variables were dummy variables for ambulatory class in the first GLM, a continuous variable for total DMDSAT score in the second GLM and dummy variables for ventilation status in the third GLM. The models were also adjusted for income class, common mental and behavioural disorders, and a dummy variable indicating additional household member with DMD to control for confounding effects. Model cost and utility data are summarised in Table 1. Given the low life expectancy in DMD and the fact that our estimates of informal care costs, caregiver indirect costs and caregiver loss in HR-QOL only concern the primary caregiver (e.g. one parent), we assumed that all patients had at least one caregiver for the duration of the simulation (while alive). No other UK cost of illness or utility estimates for patients with DMD were identified in the literature.

\subsection{Perspective of Analysis}

Our base-case scenario was executed from a healthcare perspective including direct medical costs, excluding informal care costs, as well as the impact of the disease on patient HR-QOL. In addition, we analysed a scenario from the societal perspective comprising all costs (i.e. direct medical and non-medical, including informal care costs, and indirect costs) as well as the impact on patient and primary caregiver HR-QOL. We assumed an annual treatment cost of $£ 100,000$ per patient per year (similar to some currently marketed orphan drugs [23]).

\subsection{Mortality Data}

The median life expectancy among patients with DMD receiving care in accordance with current clinical guidelines (e.g. with ventilation support) has been estimated at 25 years in the UK [8], with excess mortality almost exclusively affecting patients older than 18 years of age. In the absence of data on mean survival, we assumed that $50 \%$ of patients would die by an age of 25 years (i.e. within 7 years following their eighteenth birthday). This corresponds to an annual probability of dying of $1-(1-$ $0.5)^{(1 / 7)}=0.09$. For patients surviving to an age of 35 years, we assumed an exponential increase in mortality of $15 \%$ per annum so that no patients survived beyond their fifth decade, in line with our clinical experience and existing evidence. For patients younger than 18 years of age, we applied age-specific UK male general population mortality rates from the UK Office for National Statistics [24]. A graphical representation of the applied mortality rate is shown in Fig. 2 (light grey areas). 
Table 1 Model costs (in 2015 Great British pounds) and utility data

\begin{tabular}{|c|c|c|c|c|c|c|c|}
\hline & \multicolumn{2}{|l|}{ Direct costs } & \multicolumn{2}{|c|}{ Indirect (productivity) $\operatorname{costs}^{\mathrm{c}}$} & \multirow[t]{2}{*}{ Total cost } & \multicolumn{2}{|l|}{ Utilities } \\
\hline & Medical $^{\mathrm{a}}$ & Non-medical $^{\mathrm{b}}$ & Patient & Caregiver & & Patient & Caregiver \\
\hline \multicolumn{8}{|l|}{ Model I (DMDSAT) } \\
\hline Initial score ${ }^{\mathrm{e}}$ & $8340(830)$ & $9120(860)$ & $\begin{array}{l}14,230 \\
(1540)^{\mathrm{d}}\end{array}$ & $6360(740)$ & $23,870(1580)$ & $0.879(0.037)$ & $0.862(0.016)$ \\
\hline $\begin{array}{l}\text { Per lost score } \\
\text { (multiplier) }\end{array}$ & $1.057(1.005)$ & $1.04(1.006)$ & NA & $1.037(1.006)$ & $1.053(1.003)$ & $0.905(1.003)$ & $0.995(1.001)$ \\
\hline \multicolumn{8}{|c|}{ Model II (ambulatory status) } \\
\hline Early ambulatory & $10,670(140)$ & $9740(50)$ & $0(0)$ & $7180(190)$ & $27,590(350)$ & $0.699(0.036)$ & $0.858(0.017)$ \\
\hline Late ambulatory & $11,190(100)$ & $11,420(50)$ & $0(0)$ & $8340(150)$ & $30,950(260)$ & $0.607(0.029)$ & $0.839(0.017)$ \\
\hline Early non-ambulatory & $16,490(290)$ & $17,860(110)$ & $0(0)$ & $12,810(370)$ & $47,160(710)$ & $0.224(0.014)$ & $0.784(0.021)$ \\
\hline Late non-ambulatory & $27,590(340)$ & $16,810(90)$ & $\begin{array}{l}14,230 \\
(1540)^{\mathrm{d}}\end{array}$ & $11,240(260)$ & $66,720(1600)$ & $0.146(0.010)$ & $0.810(0.018)$ \\
\hline \multicolumn{8}{|c|}{ Model III (ventilation status) } \\
\hline None & $11,520(60)$ & $12,660(60)$ & $\begin{array}{l}14,230 \\
(1540)^{\mathrm{d}}\end{array}$ & $9160(120)$ & $34,520(440)$ & $0.518(0.027)$ & $0.837(0.014)$ \\
\hline Night-time & $31,710(590)$ & $14,610(240)$ & $\begin{array}{l}14,230 \\
(1540)^{\mathrm{d}}\end{array}$ & $10,490(420)$ & $61,490(2600)$ & $0.129(0.017)$ & $0.775(0.030)$ \\
\hline Day- and night-time & $36,390(840)$ & $15,500(190)$ & $\begin{array}{l}14,230 \\
(1540)^{\mathrm{d}}\end{array}$ & $12,860(640)$ & $83,250(2210)$ & $0.051(0.010)$ & $0.774(0.033)$ \\
\hline
\end{tabular}

Data are presented as mean (standard error) (costs roundest to the nearest ten). Source cost estimates were converted from US dollars to Great British pounds using an exchange rate of 0.634 and inflated from 2012 to 2015 values using consumer price data from the Organisation for Economic Co-operation and Development (OECD). Patient utilities (ranging from $0=$ dead to $1=$ perfect health) were obtained from the Health Utilities Index Questionnaire and caregiver utilities from the EuroQol EQ-5D-3L

DMDSAT Duchenne muscular dystrophy Functional Ability Self-Assessment Tool, NA not applicable

${ }^{a}$ Hospital admissions, emergency care, respite care, visits to physicians and other healthcare practitioners (i.e. nurses, general practitioners, specialist physicians, psychologists, therapists, physiotherapists, occupational therapists, care coordinators/care advisors, dentists, dietitians/ nutritionists and speech/language/swallowing therapists), tests and assessments, medications, medical aids, devices and investments, and community services (e.g. home help and personal assistants)

${ }^{\mathrm{b}}$ Non-medical aids, devices, and investments and cost associated with informal care (see Landfeldt et al. [10] for details)

c Valued according to the human capital approach at the cost of employment

${ }^{\mathrm{d}}$ Mean per-patient annual indirect cost for patients 18 years of age or older. Total cost may not equal the sum of total direct and indirect costs since not all patients in the specified strata accrue indirect costs (because, for example, they are $<18$ years old, attend university or are employed) and because of rounding

e Patients start the model simulation at a DMDSAT score of 23 (i.e. at full functional ability)

\subsection{Model Outcomes}

Model outcomes comprised total lifetime costs, number of life-years, and number of quality-adjusted life-years (QALYs). Lifetime cost and QALY estimates were used to calculate the incremental cost $(\Delta \mathrm{C})$ per incremental QALY $(\Delta E)$, known as the incremental cost-effectiveness ratio (ICER) $(\Delta C / \Delta E)$. Costs and QALYs were discounted at $3.5 \%$. Cost results are presented in 2015 Great British pounds $(\mathfrak{E})$, rounded to the nearest ten.

\subsection{Sensitivity Analysis}

We conducted deterministic one-way scenario analysis investigating the impact (from a healthcare perspective) of assuming different discount rates, starting treatment at 10 years of age, different treatment durations and efficacy on mortality. In addition, to help understand to which variables the ICER was most sensitive, and thereby identity which input data are most important for the different model frameworks, we ran deterministic sensitivity analysis in which key model parameters were altered (one-way) by $\pm 50 \%$.

\subsection{Model Validation}

The models were validated as suggested in the Assessment of the Validation Status of Health-Economic decision models (AdViSHE) tool [25]. Face validation of the appropriateness of the conceptual models (in terms of, 


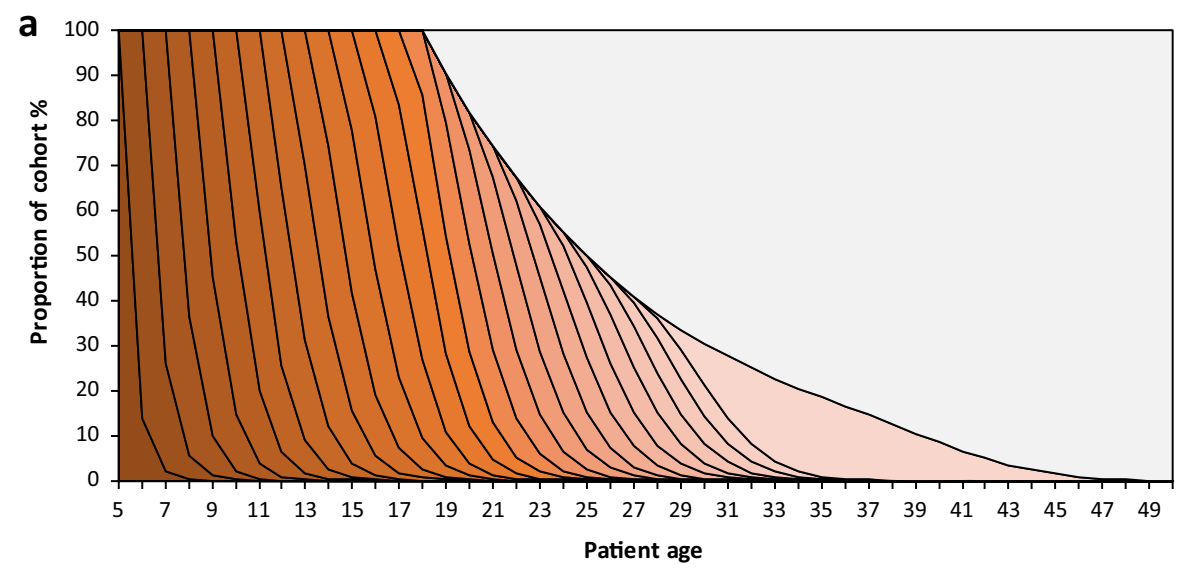

\begin{tabular}{|c|c|c|}
\hline \multicolumn{3}{|c|}{ States (DMDSAT score) } \\
\hline$\square$ Dead & $\square 0$ & $\square 1$ \\
\hline$\square 2$ & $\square 3$ & $\square 4$ \\
\hline$\square 5$ & $\square 6$ & $\square 7$ \\
\hline$\square 8$ & $\square 9$ & $\square 10$ \\
\hline$\square 11$ & $\square 12$ & $\square 13$ \\
\hline 14 & $\square 15$ & $\square 16$ \\
\hline 17 & $\square 18$ & $\square 19$ \\
\hline 20 & $\square 21$ & $\square 22$ \\
\hline
\end{tabular}

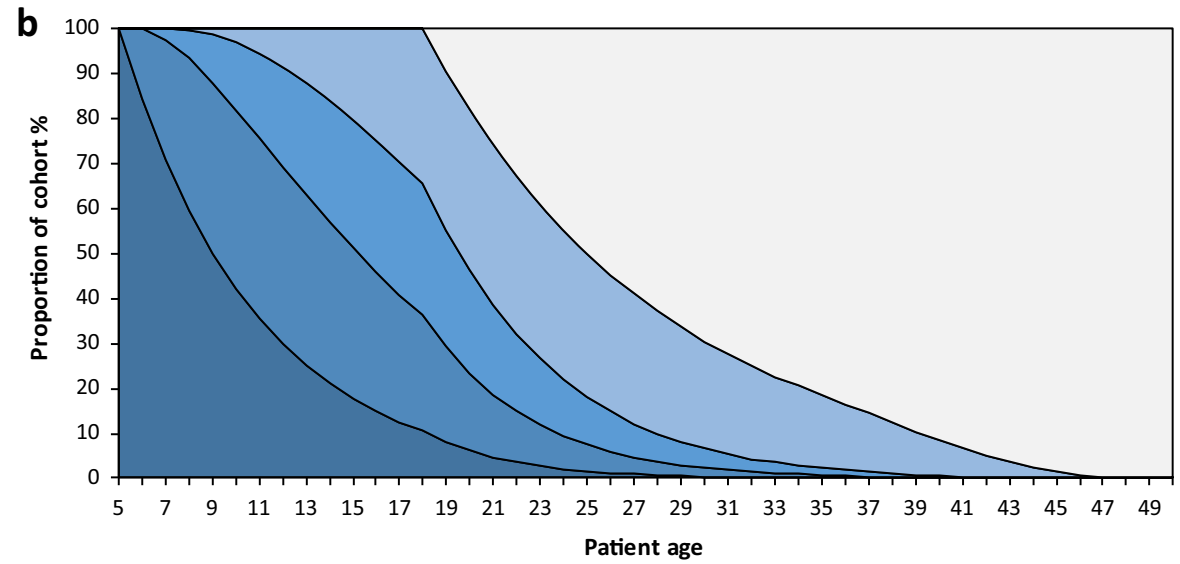

States (ambulatory class) $\square$ Dead

$\square$ Late non-ambulatory

$\square$ Early non-ambulatory

口 Late ambulatory

口 Early ambulatory

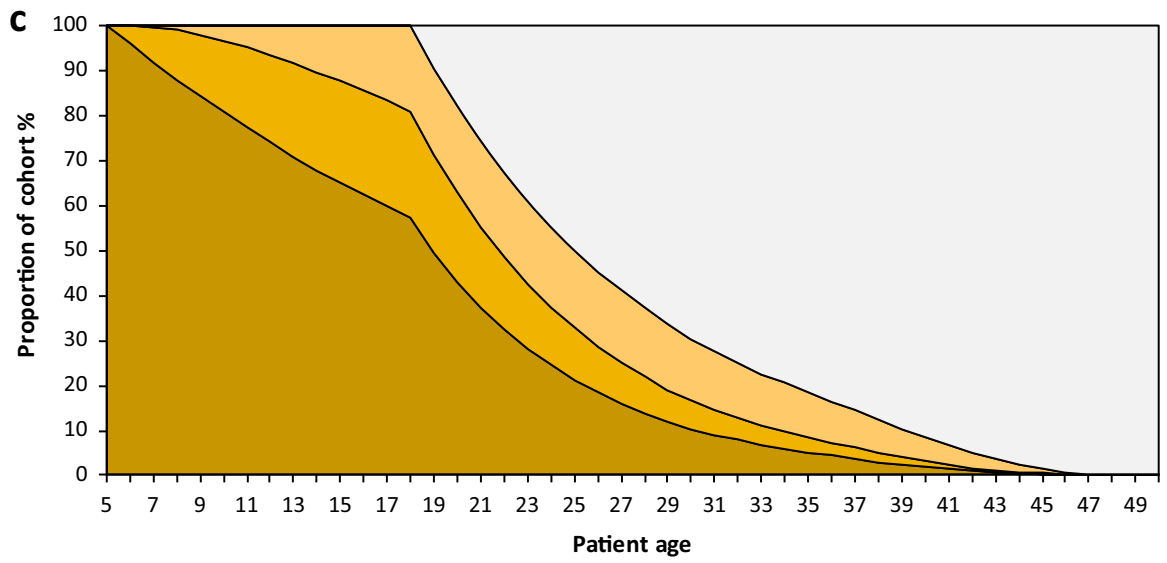

States (ventilation support)

$\square$ Dead

$\square$ Day- and night-time

$\square$ Night-time

$\square$ None

Fig. 2 Markov trace for the standard of care arm in model I (a), model II (b) and model III (c). The break at 18 years of age is caused by the introduction of Duchenne muscular dystrophy-specific mortality (see Sect. 2 for details). DMDSAT Duchenne muscular dystrophy Functional Ability Self-Assessment Tool

transition probabilities to 0 and 1 , respectively and turning off specific costs and utility components as well as mortality. No previous models of DMD were identified in the literature and cross-validation testing was therefore not possible. However, one model of a subtype of DMD has been described as part of a NICE appraisal [26]. 


\section{Results}

\subsection{Model Validation}

All three models were judged to have good validity with regards to the appropriateness of the choice of modelling technique, conceptual representation of the disease, model input data and model outcomes. Markov traces for the placebo arms (Fig. 2) showed that the simulated cohorts transitioned across model states in agreement with the input data. Specifically, regarding mortality, when starting the simulation at an age of 5 years, $50 \%$ of patients survived until an age of 25 years, the estimated number of (undiscounted) life-years was 23 and only a small proportion survived beyond an age of 40 years $(<7 \%)$, with no patients surviving into their fifth decade. In the placebo arm, in model I, exactly $86 \%$ of the living cohort transitioned between model states each cycle throughout the simulation. In model II, patients remained in each modelled ambulatory class for 4 years, on average, and in model III exactly 4 and $9 \%$ of the cohort transitioned from the 'none' to 'night-time' and 'night-time' to 'day- and night-time' ventilation states, respectively, each cycle. Extreme value and unit testing revealed no errors with regards to the mathematical implementation of the model.

\subsection{Base-Case and Sensitivity Analysis Results}

Results for the healthcare and societal perspective scenario for each model are presented in Table 2. Table 3 presents outcomes of the one-way scenario analysis. Results from the sensitivity analysis in which key model parameters were altered one-way by $\pm 50 \%$ showed that the ICER, in addition to disease progression probabilities and annual drug cost, was most sensitive to initial patient utility value in model I, early ambulatory patient utility in model II, and caregiver utility for non-ventilated patients in model III. Additional sensitivity analysis results provided in the Electronic Supplementary Material Appendix.

\section{Discussion}

This study is the first to present a decision-analytic model framework for the economic evaluation of treatments for DMD based on the DMDSAT, and to our knowledge is the first to present and compare any models for the assessment of the cost effectiveness of treatments for DMD. However, one model of the disease has been described as part of a NICE appraisal for ataluren [26], a treatment targeting a subgroup of patients with DMD caused by nonsense mutation in the dystrophin gene. This model, which was based on our previously published cost and utility data, comprised a total of five states (in addition to an absorbing state for dead): (1) ambulatory; (2) non-ambulatory; (3) non-ambulatory with scoliosis; (4) non-ambulatory with ventilation support; and (5) non-ambulatory with ventilation support and scoliosis. Comparing our frameworks with the ataluren model, the following may be noted. First, in the ataluren model, differences between arms (i.e. ataluren vs. placebo) related only to time to non-ambulation based on outcomes from the 6-Minute Walk Test (6MWT) the primary trial efficacy endpoint, with identical transition probabilities for subsequent states. As a result, the mean costs associated with scoliosis and ventilation support could have been added to the non-ambulatory state (accounting for assumed differences between arms), and the model therefore essentially only comprised two states: ambulatory and non-ambulatory (in addition to dead). Accordingly, the ataluren model may be regarded as a less granular version of model II (which was based on four ambulatory classes). Second, as noted in Sect. 2, modelling specific complications associated with DMD, or adjusting total cost and utility estimates for disease-related events (e.g. scoliosis or ventilation support) at the cohort or patient level is not straightforward due to lack of data. In the ataluren model, scoliosis (but not ventilation support) was assumed to be associated with an additional cost and patient utility loss. However, no adjustments were made to the costs and utilities that were assigned to the non-scoliosis non-ventilation support model states, despite the fact that these estimates were derived for a sample of patients of which a substantial proportion in fact had scoliosis and/or received ventilation support. Thus, our DMD cost and utility data appear to have been incorrectly implemented into the structure of the ataluren model. Third, in the ataluren evaluation, assumptions regarding mortality seem to be in poor agreement with the current body of evidence. Specifically, from the Markov traces, it appears as if patients receiving placebo had a median survival of about 33 years, with a substantial proportion (30\% of patients on average) surviving beyond 40 years as well as 50 years of age $(15 \%)$. In contrast, our representation of mortality in DMD (light grey areas in Fig. 2) was found to be in good agreement with current evidence and our clinical expectations given current SoC. Specifically, our simulations yielded a median survival of 25 years, with only a small proportion of patients surviving beyond 40 years and no patients beyond 50 years of age, on average.

Comparing estimates from the healthcare perspective analysis across the three models, our results revealed that the choice of model structure may have a considerable impact on the outcomes of economic evaluations of treatments for DMD. Specifically, results from the model based on the DMDSAT (model I) showed that the total discounted lifetime cost of DMD for patients receiving $\mathrm{SoC}$ 
Table 2 Cost effectiveness of a hypothetical treatment for Duchenne muscular dystrophy

\begin{tabular}{|c|c|c|c|c|c|c|c|c|c|}
\hline & \multicolumn{3}{|c|}{ Model I (DMDSAT) } & \multicolumn{3}{|c|}{ Model II (ambulatory status) } & \multicolumn{3}{|c|}{ Model III (ventilation status) } \\
\hline & Trt & SoC & $\Delta$ & Trt & SoC & $\Delta$ & Trt & SoC & $\Delta$ \\
\hline \multicolumn{10}{|l|}{ Cost outcomes } \\
\hline Intervention cost & $1,547,110$ & 0 & $1,547,110$ & $1,547,110$ & 0 & $1,547,110$ & $1,547,110$ & 0 & $1,547,110$ \\
\hline Direct medical costs & 190,840 & 217,510 & $-26,670$ & 221,250 & 244,120 & $-22,860$ & 262,050 & 284,640 & $-22,600$ \\
\hline Direct non-medical costs & 184,330 & 201,290 & $-16,960$ & 194,520 & 204,830 & $-10,310$ & 204,580 & 207,080 & -2500 \\
\hline Patient indirect costs & 69,000 & 69,000 & 0 & 69,000 & 69,000 & 0 & 69,000 & 69,000 & 0 \\
\hline Caregiver indirect costs & 125,850 & 136,440 & $-10,590$ & 139,490 & 145,560 & -6070 & 150,150 & 153,130 & -2980 \\
\hline \multicolumn{10}{|l|}{ Total costs $(\mathrm{C})$} \\
\hline Healthcare perspective & $1,737,960$ & 217,510 & $1,520,450$ & $1,768,370$ & 244,120 & $1,524,250$ & $1,809,160$ & 284,640 & $1,524,520$ \\
\hline Societal perspective & $2,117,140$ & 624,240 & $1,492,900$ & $2,171,380$ & 663,500 & $1,507,870$ & $2,232,890$ & 713,840 & $1,519,040$ \\
\hline \multicolumn{10}{|l|}{ Effect outcomes (E) } \\
\hline Patient QALYs & 8.13 & 7.07 & 1.05 & 7.96 & 7.17 & 0.79 & 6.39 & 5.96 & 0.43 \\
\hline Caregiver QALYs & 12.93 & 12.80 & 0.12 & 12.89 & 12.82 & 0.07 & 12.72 & 12.66 & 0.06 \\
\hline \multicolumn{10}{|l|}{$\operatorname{ICER}(\Delta \mathrm{C} / \Delta \mathrm{E})$} \\
\hline Healthcare perspective & & & $1,442,710$ & & & $1,939,590$ & & & $3,574,770$ \\
\hline Societal perspective & & & $1,266,510$ & & & $1,760,650$ & & & $3,121,890$ \\
\hline
\end{tabular}

The ICER was calculated as the difference in total costs $(\Delta \mathrm{C})$ divided by the difference in total QALY gains $(\Delta \mathrm{E})$. Cost results are reported in 2015 Great British pounds $(\mathfrak{E})$ rounded to the nearest ten. Costs and effects were discounted at $3.5 \%$

$\triangle$ difference, ICER incremental cost-effectiveness ratio, $Q A L Y s$ quality-adjusted life-years, SoC standard of care, Trt treatment

Table 3 Sensitivity analysis results (treatment versus standard of care, healthcare perspective)

\begin{tabular}{|c|c|c|c|c|c|c|c|c|c|}
\hline \multirow[t]{2}{*}{ Scenario } & \multicolumn{3}{|c|}{ Model I (DMDSAT) } & \multicolumn{3}{|c|}{ Model II (ambulatory status) } & \multicolumn{3}{|c|}{ Model III (ventilation status) } \\
\hline & 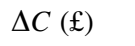 & $\Delta E$ & 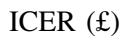 & 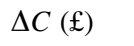 & $\Delta E$ & 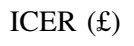 & 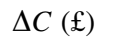 & $\Delta E$ & $\operatorname{ICER}(£)$ \\
\hline Base case & $1,520,450$ & 1.05 & $1,442,710$ & $1,524,250$ & 0.79 & $1,939,590$ & $1,524,520$ & 0.43 & $3,574,770$ \\
\hline Discount rate $0 \%^{\mathrm{a}}$ & $2,212,630$ & 1.62 & $1,369,720$ & $2,222,920$ & 1.25 & $1,774,820$ & $2,223,900$ & 0.72 & $3,088,290$ \\
\hline Discount rate $5 \%^{\mathrm{a}}$ & $1,324,480$ & 0.89 & $1,485,900$ & $1,326,910$ & 0.65 & $2,031,000$ & $1,326,920$ & 0.35 & $3,811,080$ \\
\hline Starting treatment at 10 years of age & $1,269,530$ & 0.83 & $1,521,070$ & $1,271,430$ & 0.60 & $2,131,650$ & $1,271,040$ & 0.31 & $4,064,600$ \\
\hline 5-Year treatment & $1,538,770$ & 0.46 & $3,313,550$ & 459,060 & 0.31 & $1,499,270$ & 458,130 & 0.16 & $2,823,400$ \\
\hline 10-Year treatment & $1,530,870$ & 0.80 & $1,910,320$ & 843,150 & 0.57 & $1,479,360$ & 842,630 & 0.30 & $2,844,400$ \\
\hline Efficacy on mortality ${ }^{b}$ & $1,630,980$ & 1.23 & $1,324,740$ & $1,635,930$ & 1.03 & $1,590,120$ & $1,638,360$ & 0.68 & $2,394,430$ \\
\hline
\end{tabular}

Cost results in 2015 Great British pounds ( $(\mathfrak{)})$ rounded to the nearest ten

$\triangle C$ difference in total costs, $\triangle E$ difference in total QALY gains, DMDSAT Duchenne muscular dystrophy Functional Ability Self-Assessment Tool, ICER incremental cost-effectiveness ratio, $Q A L Y$ quality-adjusted life-year

${ }^{a}$ Rate applied to costs and QALYs

b Assuming a $25 \%$ reduction in the Duchenne muscular dystrophy-specific mortality

was $£ 217,510$ when only considering direct medical costs. The introduction of the hypothetical treatment, which was assumed to delay disease progression by $25 \%$, resulted in a patient QALY gain of 1.05 due to maintained HR-QOL, a reduction in direct medical costs of $£ 26,670$ and an ICER of $£ 1,442,710$ ( $£ 1,520,450 / 1.05)$ assuming an annual drug cost of $£ 100,000$ (equal to $£ 1,547,110$ during the lifetime of the patient). Corresponding ICERs from models II and III were markedly higher due to the notably less granular structures yielding a lower number of patient QALYs gained and lower cost reductions. Accordingly, as costs and utilities are markedly different across the disease progression sequence in DMD - which for some patients spans 30 or even 40 years with current care-due to heterogeneous 
presentation of the disease, these findings suggest that grouping patients into a low number of states representing stages of disease (i.e. four in model II and three in model III, compared with 24 states in model I) may result in poor sensitivity with respect to costs and utilities due to averaging and, as a consequence, a crude simulation of DMD as interpreted through the health economics of the disease.

Comparing our results across analysis perspectives, the inclusion of caregiver HR-QOL resulted in relatively modest QALY gains, whereas accounting for costs beyond those directly attributed to formal care had a considerable impact on the evaluation results. Specifically, in model I the mean lifetime cost for patients receiving $\mathrm{SoC}$ was estimated at $£ 624,240$ from the societal perspective, almost three times as high as the lifetime cost estimate from the healthcare perspective. This result reflects the fact that long-term care of patients with DMD usually is provided at home by family caregivers, rather than in clinics by healthcare professionals. In fact, as reported in our previous work, informal care and indirect costs together account for approximately $47 \%$ of total costs of illness in the UK [10]. In the context of HTA, this finding emphasises the importance of considering all costs, not only those attributed to formal care, in evaluations of treatments for chronic childhood diseases such as DMD to allow for a meaningful appraisal of treatment benefits. Accounting for differences in direct medical, direct non-medical, and patient and caregiver indirect costs resulted in an ICER of $£ 1,266,510$ $(£ 1,492,900 / 1.17)$ in model I, $£ 1,760,650$ ( $£ 1,507,870 /$ $0.86)$ in model II and $£ 3,121,890(£ 1,519,040 / 0.49)$ in model III, corresponding to a difference of between $£ 176,200$ and $£ 452,880$ across frameworks compared with the healthcare perspective.

In addition to the merits mentioned earlier regarding the model based on the DMDSAT compared with models II and III, there are several potential advantages of including the DMDSAT in development programmes of new health technologies for DMD. First, the DMDSAT is currently the only rating scale designed to measure progression in DMD across the entire trajectory of disease. Given that the DMDSAT is an interval instrument, which allows for meaningful measurement of changes in total scores irrespective of patients' current disease stage, this would be expected to greatly facilitate recruitment of patients to trials, as well as to measure efficacy for different levels of disease severity (which would allow modelling of nonlinear efficacy levels). Second, including the DMDSAT in development programmes of new treatments (e.g. as a secondary endpoint in phase III trials) would help avoid difficulties in translating efficacy measures from, for example, the 6MWT to model transition probabilities. This is of particular importance for more advanced stages of the disease considering that the 6MWT is not applicable to non-ambulatory patients. Third, the DMDSAT scores have been mapped to cost and utility data, which may greatly facilitate implementation and execution of economic evaluations.

The assumptions included in our model are a source of potential limitations. For example, longitudinal natural history data are not available for the DMDSAT and we therefore based the progression in the SoC arm on our cross-sectional data. Mean life expectancy for patients with DMD receiving care in line with current clinical guidelines is also lacking and we therefore made assumptions based on available median estimates and our clinical experience. With respect to these data gaps, outcomes from ongoing clinical trials and epidemiological studies in DMD would be expected to help strengthen the robustness of the model input data and thereby improve the validity of the proposed model frameworks. An additional limitation concerns the fact that our input data were identified in a targeted literature review, as opposed to a full systemic review.

\section{Conclusion}

We present a first version of a model for the economic evaluation of treatments for DMD based on the DMDSAT, as well as two alternative frameworks based on conventional staging of disease progression. Our findings should be helpful to inform HTAs and health economic programmes of future treatments for DMD.

\section{Compliance with Ethical Standards}

\section{Funding None.}

Conflict of interest Mr Landfeldt, Dr Alfredsson, Dr Straub, Dr Lochmüller, Dr Bushby and Dr Lindgren report no conflicts of interest.

Contributions Mr EL designed the mathematical models with input from Dr PL, Dr KB and Dr HL. Mr EL developed the mathematical models and executed all analyses. Mr EL led the interpretation of findings together with Dr PL, Dr KB and Dr HL with input from the other authors. Mr EL drafted the manuscript. All authors reviewed the manuscript and approved the decision to submit for publication.

Open Access This article is distributed under the terms of the Creative Commons Attribution-NonCommercial 4.0 International License (http://creativecommons.org/licenses/by-nc/4.0/), which permits any noncommercial use, distribution, and reproduction in any medium, provided you give appropriate credit to the original author(s) and the source, provide a link to the Creative Commons license, and indicate if changes were made. 


\section{References}

1. Drummond M, Towse A. Orphan drugs policies: a suitable case for treatment. Eur J Health Econ. 2014;15:335-40.

2. Drummond M, Wilson DA, Kanavos P, Ubel P, Rovira J. Assessing the economic challenges posed by orphan drugs. Int $\mathbf{J}$ Technol Assess Health Care. 2007;23:36-42.

3. Simoens S, Picavet E, Dooms M, Cassiman D, Morel T. Costeffectiveness assessment of orphan drugs: a scientific and political conundrum. Appl Health Econ Health Policy. 2013;11:1-3.

4. Braun MM, Farag-El-Massah S, Xu K, Coté TR. Emergence of orphan drugs in the United States: a quantitative assessment of the first 25 years. Nat Rev Drug Discov. 2010;9:519-22.

5. Hughes-Wilson W, Palma A, Schuurman A, Simoens S. Paying for the orphan drug system: break or bend? Is it time for a new evaluation system for payers in Europe to take account of new rare disease treatments? Orphanet J Rare Dis. 2012;7:74.

6. Gammie T, Lu CY, Babar ZU. Access to orphan drugs: a comprehensive review of legislations, regulations and policies in 35 countries. PLoS One. 2015;10(10):e0140002.

7. Mendell JR, Shilling C, Leslie ND, Flanigan KM, al-Dahhak R, Gastier-Foster J, et al. Evidence-based path to newborn screening for Duchenne muscular dystrophy. Ann Neurol 2012;71:304-313.

8. Eagle M, Bourke J, Bullock R, Gibson M, Mehta J, Giddings D, et al. Managing Duchenne muscular dystrophy-the additive effect of spinal surgery and home nocturnal ventilation in improving survival. Neuromuscul Disord. 2007;17:470-5.

9. Bushby K, Finkel R, Birnkrant DJ, Case LE, Clemens PR, Cripe L, DMD Care Considerations Working Group, et al. Diagnosis and management of Duchenne muscular dystrophy, part 1: diagnosis, and pharmacological and psychosocial management. Lancet Neurol. 2010;9:77-93.

10. Landfeldt E, Lindgren P, Bell C, Schmitt C, Guglieri M, Straub $\mathrm{V}$, et al. The burden of Duchenne muscular dystrophy: an international, cross-sectional study. Neurology. 2014;83:529-36.

11. Landfeldt E, Lindgren P, Bell C, Guglieri M, Straub V, Lochmüller $\mathrm{H}$, et al. Health-related quality of life in patients with Duchenne muscular dystrophy: a multinational, cross-sectional study. Dev Med Child Neurol. 2016;58(5):508-15.

12. Landfeldt E, Lindgren P, Bell CF, Guglieri M, Straub V, Lochmüller $\mathrm{H}$, et al. Quantifying the burden of caregiving in Duchenne muscular dystrophy. J Neurol. 2016;263(5):906-15.

13. Landfeldt E, Mayhew A, Eagle M, Lindgren P, Bell CF, Guglieri $\mathrm{M}$, et al. Development and psychometric analysis of the Duchenne muscular dystrophy Functional Ability Self-Assessment Tool (DMDSAT). Neuromuscul Disord. 2015;25(12):937-44.
14. Caro JJ, Briggs AH, Siebert U, Kuntz KM, ISPOR-SMDM Modeling Good Research Practices Task Force. Modeling good research practices-overview: a report of the ISPOR-SMDM Modeling Good Research Practices Task Force-1. Value Health. 2012;15:796-803.

15. Wang RT, Silverstein Fadlon CA, Ulm JW, Jankovic I, Eskin A, $\mathrm{Lu} \mathrm{A}$, et al. Online self-report data for Duchenne muscular dystrophy confirms natural history and can be used to assess for therapeutic benefits. PLoS Curr. 2014. doi:10.1371/currents.md. e1e8f2be7c949f9ffe81ec6fca1cce6a.

16. Ricotti V, Ridout DA, Scott E, Quinlivan R, Robb SA, Manzur AY, et al. NorthStar Clinical Network. Long-term benefits and adverse effects of intermittent versus daily glucocorticoids in boys with Duchenne muscular dystrophy. J Neurol Neurosurg Psychiatry. 2013;84(6):698-705.

17. Humbertclaude V, Hamroun D, Bezzou K, Bérard C, BoespflugTanguy $\mathrm{O}$, Bommelaer $\mathrm{C}$, et al. Motor and respiratory heterogeneity in Duchenne patients: implication for clinical trials. Eur J Paediatr Neurol. 2012;16(2):149-60.

18. Horsman J, Furlong W, Feeny D, Torrance G. The Health Utilities Index (HUI): concepts, measurement properties and applications. Health Qual Life Outcomes. 2003;1:54.

19. Feeny DH, Furlong WJ, Torrance GW, Goldsmith $\mathrm{CH}$, Zhu Z, DePauw S, et al. Multiattribute and single-attribute utility functions for the Health Utilities Index Mark 3 system. Med Care. 2002;40:113-28.

20. EuroQol Group. EuroQol-a new facility for the measurement of health-related quality of life. Health Policy. 1990;16(3):199-208.

21. Dolan P. Modeling valuations for EuroQol health states. Med Care. 1997;35:1095-108.

22. Gregori D, Petrinco M, Bo S, Desideri A, Merletti F, Pagano E. Regression models for analyzing costs and their determinants in health care: an introductory review. Int $\mathrm{J}$ Qual Health Care. 2011;23(3):331-41.

23. Picavet E, Morel T, Cassiman D, Simoens S. Shining a light in the black box of orphan drug pricing. Orphanet J Rare Dis. 2014;9:62.

24. Office for National Statistics. Datasets and reference tables. http://www.ons.gov.uk. Accessed 15 Feb 2015.

25. Vemer P, Corro Ramos I, van Voorn GA, Al MJ, Feenstra TL. AdViSHE: a validation-assessment tool of health-economic models for decision makers and model users. Pharmacoeconomics. 2016;34(4):349-61.

26. NICE guidance for ataluren. https://www.nice.org.uk/guidance/ hst3?unlid=7891072072016831154657. Accessed 15 Apr 2016. 